2014

\title{
Using Unnatural Amino Acid Mutagenesis To Probe the Regulation of PRMT1
}

\author{
Heather L. Rust \\ Venkataraman Subramanian \\ Paul R. Thompson \\ Douglas D. Young \\ College of William and Mary
}

Follow this and additional works at: https://scholarworks.wm.edu/aspubs

\section{Recommended Citation}

Rust, H. L., Subramanian, V., West, G. M., Young, D. D., Schultz, P. G., \& Thompson, P. R. (2014). Using unnatural amino acid mutagenesis to probe the regulation of PRMT1. ACS chemical biology, 9(3), 649-655. accepted for inclusion in Arts \& Sciences Articles by an authorized administrator of W\&M ScholarWorks. For more information, please contact scholarworks@wm.edu. 


\title{
Using Unnatural Amino Acid Mutagenesis To Probe the Regulation of PRMT1
}

\author{
Heather L. Rust, ${ }^{\dagger}$ Venkataraman Subramanian, ${ }^{\dagger}$ Graham M. West, ${ }^{\ddagger}$, Douglas D. Young, \\ Peter G. Schultz, ${ }^{\perp}$ and Paul R. Thompson ${ }^{*}{ }^{\dagger}$
}

Departments of ${ }^{\dagger}$ Chemistry, ${ }^{\ddagger}$ Mass Spectrometry and Proteomics, and ${ }^{\S}$ Molecular Therapeutics, The Scripps Research Institute, 130 Scripps Way, Jupiter, Florida 33458, United States

"Department of Chemistry, The College of William \& Mary, P.O. Box 8795, Williamsburg, Virginia 23185, United States

${ }^{\perp}$ Department of Chemistry, The Scripps Research Institute, 10550 North Torrey Pines Road, La Jolla, California 92037, United States

\section{Supporting Information}

ABSTRACT: Protein arginine methyltransferase 1 (PRMT1)dependent methylation contributes to the onset and progression of numerous diseases (e.g., cancer, heart disease, ALS); however, the regulatory mechanisms that control PRMT1 activity are relatively unexplored. We therefore set out to decipher how phosphorylation regulates PRMT1 activity. Curated mass spectrometry data identified Tyr291, a residue adjacent to the conserved THW loop, as being phosphorylated. Natural and unnatural amino acid mutagenesis, including the incorporation of $p$-carboxymethyl-Lphenylalanine $(p \mathrm{CmF})$ as a phosphotyrosine mimic, were used to show that Tyr291 phosphorylation alters the substrate specificity of PRMT1. Additionally, p-benzoyl-L-phenylalanine $(p \mathrm{BpF})$ was incorporated at the Tyr291 position, and crosslinking experiments with K562 cell extracts identified several proteins (e.g., hnRNPA1 and hnRNP H3) that bind specifically to this site. Moreover, we also demonstrate that Tyr291 phosphorylation impairs PRMT1's ability to bind and methylate both proteins. In total, these studies demonstrate that Tyr291 phosphorylation alters both PRMT1 substrate specificity and protein-protein interactions.
$\mathrm{P}$ ost-translational modifications (PTMs) play a pivotal role in the cell by adding additional regulatory layers. The methylation of protein-embedded arginines is one example, and this PTM regulates gene transcription, RNA splicing, and cell proliferation. ${ }^{1,2}$ Arginine methylation is catalyzed by the protein arginine methyltransferases (PRMT), which produce $\omega$ monomethylarginine ( $\omega$-MMA) by transferring a methyl group from $S$-adenosyl-methionine (SAM) to the guanidinium moiety of an arginine. This modified residue can then be further methylated to produce asymmetrically dimethylated arginine (ADMA) or symmetrically dimethylated arginine (SDMA).

Since arginine methylation regulates such a diverse array of cellular processes, these enzymes must be regulated. In fact, phosphorylation is known to control the activity of both PRMT4 (CARM1) ${ }^{3-5}$ and PRMT5. ${ }^{6}$ For example, PRMT4 is phosphorylated at Ser217, and this modification diminishes its methyltransferase activity in vitro and abrogates its coactivator function in vivo. ${ }^{3}$ Ser217 phosphorylation likely disrupts an $\mathrm{H}$ bond between the side chain hydroxyl and backbone carbonyl of Tyr154, and as a result, the enzyme no longer binds SAM. $^{3,7-9}$ PRMT5 is also phosphorylated in vivo at three conserved tyrosines (i.e., Tyr297, Tyr304, and Tyr307). Phosphorylation of these residues, which is catalyzed by constitutively active Janus kinase 2 (JAK2) mutants, ${ }^{6}$ inhibits its association with MEP50, resulting in decreased PRMT5 activity. ${ }^{6}$

In contrast to PRMTs 4 and 5, no studies have shown that phosphorylation alters PRMT1 activity. In fact, information on how PTMs regulate PRMT1 activity is extremely limited. Given that PRMT1 shows high in vitro activity, has the widest tissue distribution and highest expression levels, and is thought to be responsible for the generation of $\sim 85 \%$ of all the methylated arginine residues found in vivo, ${ }^{10}$ this isozyme is likely subject to multiple layers of regulation, including phosphorylation or another PTM. Since PRMT1-dependent methylation contributes to the onset and progression of a range of diseases (e.g., cancer, heart disease, and ALS), ${ }^{1,2}$ a deeper understanding of its regulation is required.

Received: November 19, 2013

Accepted: December 20, 2013

Published: December 20, 2013 

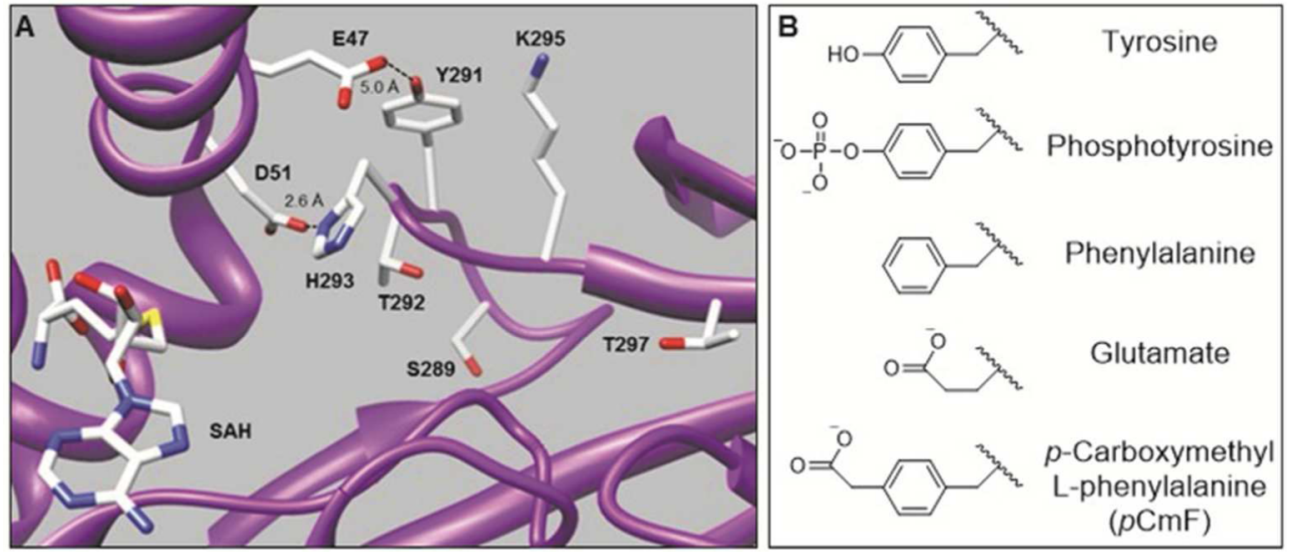

Figure 1. Structure of PRMT1. (A) Structure of PRMT1-SAH complex highlighting residues surrounding the THW loop. (B) Structures of tyrosine and phosphotyrosine analogue used in these studies. This figure was prepared with UCSF Chimera using the coordinates from PRMT1 (PDB id 1ORI).

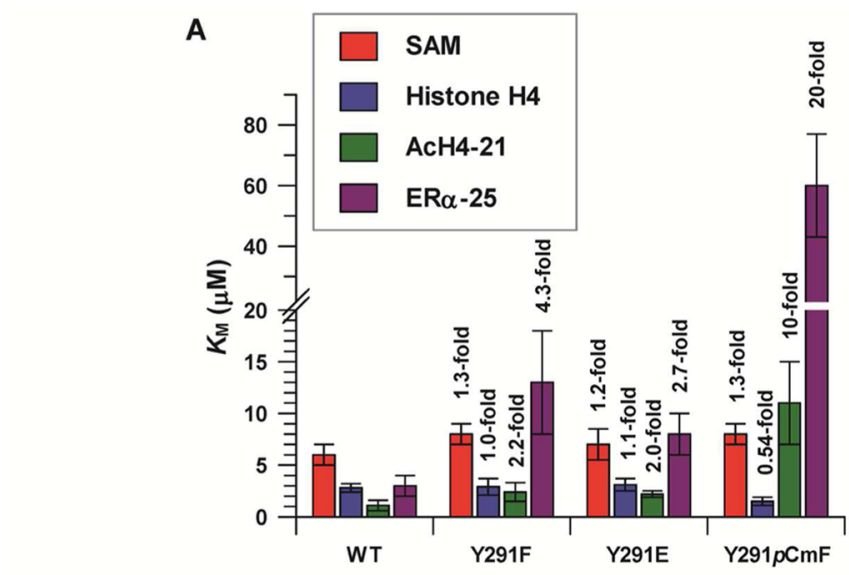

B

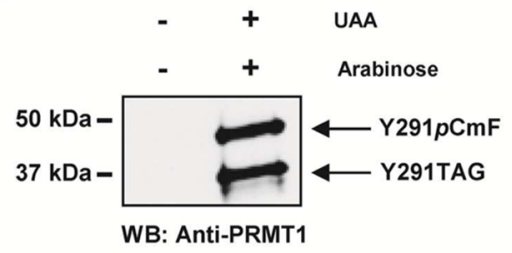

C

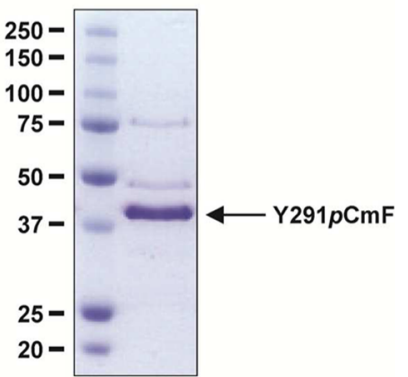

Figure 2. Incorporation of $p \mathrm{CmF}$ into the Y291TAG PRMT1 mutant and kinetic analysis of PRMT1 mutants. (A) Comparison of kinetic parameters of PRMT1 and mutants with SAM, Histone H4, AcH4-21, and ER $\alpha-25$. (B) Western blot analysis demonstrating the expression of the full length protein, with the incorporation of $p \mathrm{CmF}$ at $\mathrm{Y} 291$, as well as the truncated form. (C) Coomasie-stained gel displaying the purified Y291pCmF mutant. The yield was $1 \mathrm{mg} / \mathrm{L}$ of cell culture. Purity is estimated to be $>90 \%$.

To address how phosphorylation regulates PRMT1 activity, we used PhosphoSitePlus (www.phosphosite.org) ${ }^{11}$ to identify several in vivo sites of PRMT1 phosphorylation. PhosphoSitePlus is a curated source of experimentally determined PTMs. ${ }^{11}$ From this database, we focused on Tyr291 phosphorylation because this PTM is the most frequently identified, is present in the conserved THW loop (see below), and is elevated in a number of cancer cell lines (e.g., K-562, AMO-1, and KMS27). ${ }^{11}$ On the basis of the PRMT1 structure, we hypothesized that phosphorylation would inhibit PRMT1 activity because the introduction of a negatively charged phosphate group at Tyr291 should introduce electrostatic repulsions that destabilize interactions between the Glu47-containing helix and the THW loop (Figure 1). This hypothesis was based on previous studies ${ }^{9}$ demonstrating that the stabilization of this helix (by a salt bridge between His293 and Asp51) is important for catalysis. ${ }^{9}$ Since no direct methods (e.g., site-directed mutagenesis) have been used to ascertain how Tyr291 phosphorylation regulates PRMT1 activity, we set out to determine its function. Herein, we describe the results of these efforts. 


\section{RESULTS AND DISCUSSION}

Kinetic Analysis of PRMT1 Phosphorylation Mutants. To evaluate the importance of the Tyr291 hydroxyl group, we first generated the Y291F and Y291E mutants. Kinetic studies revealed that neither mutation has a dramatic effect on the kinetic parameters for SAM, histone $\mathrm{H} 4$, as well as peptide mimics of the histone $\mathrm{H} 4$ tail region (i.e., AcH4-21) or the site of methylation in ER $\alpha$ (i.e., the ER $\alpha$-25 peptide) (Figure $2 \mathrm{~A}$ and Supplementary Tables S1-S4). Because of the size and electronic differences between glutamate and phosphotyrosine (Figure 1), we used unnatural amino acid (UAA) mutagenesis to install $p$-carboxymethyl-L-phenylalanine $(p \mathrm{CmF})$ at this position and thereby better mimic a phosphotyrosine. ${ }^{12} \mathrm{We}$ focused here on the use of unnatural amino acid mutagenesis to install $p \mathrm{CmF}$ because the central location of Tyr291 in a highly structured region of PRMT1 precluded the use of protein semisynthetic methods to install a phosphonate analogue. ${ }^{13}$

To generate the Y291pCmF mutant, the Tyr291 codon was mutated to TAG and $p \mathrm{CmF}$ was synthesized (Supplementary Scheme S1) and incorporated using a previously evolved orthogonal amber suppressor tRNA/aminoacyl-tRNA synthetase pair. ${ }^{12,14}$ Although both the full length protein (with $p \mathrm{CmF}$ incorporated) and the truncated Y291TAG mutant are expressed (Figure 2B), the truncated protein does not copurify with the Y291 $\mathrm{CmF}$ mutant (Figure $2 \mathrm{C}$ ), likely due to its inability to fold properly. Installation of $p \mathrm{CmF}$ was confirmed by tandem MS (Supplementary Figure S1 and Table S5), and none of the amino acid substitutions affected protein stability, as measured by partial proteolysis experiments (Supplementary Figure S2).

Similarly to the Y291F and Y291E mutants, the Y291pCmF mutation showed limited effects on the kinetic parameters for SAM (Figure 2A and Supplementary Table S1). Given the structure of PRMT1 and our previous studies showing that destabilization of the N-terminal helix impacts SAM binding, these results were somewhat surprising. To further investigate the role of this modification, we next determined the kinetic parameters for histone $\mathrm{H} 4$ and found that the $\mathrm{Y} 291 \mathrm{pCmF}$ mutation shows only small effects on $k_{\text {cat }}, K_{\mathrm{M}}$, and $k_{\text {cat }} / K_{\mathrm{M}}$; the effect is $\leq 2.4$-fold (Figure $2 \mathrm{~A}$ and Supplementary Supplementary Table S2). By contrast, we observed significant effects on the kinetic parameters for methylation of the AcH4-21 and ER $\alpha$-25 peptides (Figure 2A and Supplementary Table S3-S4). For example, with the AcH4-21 peptide, the $\mathrm{Y} 291 \mathrm{pCmF}$ mutant yielded a dramatic $\sim 11$-fold decrease in $k_{\text {cat }} / K_{\mathrm{M}}$ (Supplementary Table S3) with a comparable $\sim 10$-fold increase in $K_{\mathrm{M}}$. Similar results were obtained with ER $\alpha-25$ (Supplementary Table S4). Although incorporation of $p \mathrm{CmF}$ dramatically impacted $k_{\mathrm{cat}} / K_{\mathrm{M}}$, there was no effect on the processivity of the enzyme (Supplementary Figure S3). Despite the lack of a large effect with histone $\mathrm{H} 4$, which is likely due to additional interactions between the core of H4 and PRMT1, the results for the peptide substrates strongly argues in favor of the notion that Tyr291 phosphorylation can modulate the substrate specificity of PRMT1 in a substrate-specific manner. Furthermore, the presence of a second negative charge, as present in phosphotyrosine, would likely yield an even more dramatic effect.

Incorporation of $p \mathrm{BpF}$, a Photo-Cross-Linking Amino Acid. Given the variable effects of methylating protein versus peptide substrates, we also considered the possibility that Tyr291 phosphorylation may act as part of a docking site for substrates and/or regulatory proteins that act to fine-tune PRMT1 cellular activity. Therefore, $p$-benzoyl-L-phenyl-alanine $(p \mathrm{BpF})$, a known photo-cross-linker, ${ }^{15,16}$ was incorporated (Figure $3 \mathrm{~A}$ and $\mathrm{B}$ ) to facilitate the isolation of proteins that
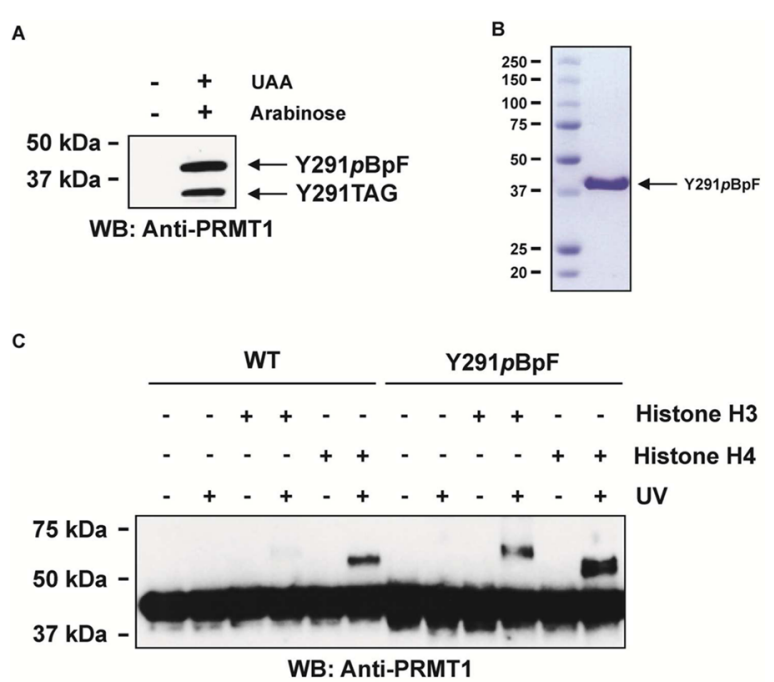

Figure 3. Incorporation of $p \mathrm{BpF}$ into Y291TAG PRMT1 mutant and cross-linking with histones. (A) Western blot analysis demonstrating the expression of the full length protein, with the incorporation of $p \mathrm{BpF}$ at $\mathrm{Y} 291$, as well as the truncated form. (B) Coomasie-stained gel displaying the purified $\mathrm{Y} 291 \mathrm{pBpF}$ mutant. The yield was $2 \mathrm{mg} / \mathrm{L}$ of cell culture. Purity is estimated to be $>90 \%$. (Cc) Cross-linking of wild type and the $\mathrm{Y} 291 \mathrm{pBpF}$ mutant with histone $\mathrm{H} 3$ and $\mathrm{H} 4$.

interact with Tyr291. This substitution has only minor effects on the kinetic parameters for SAM, histone $\mathrm{H} 4$, and the AcH421 peptide (Supplementary Tables $\mathrm{S} 1-\mathrm{S} 3$ ). Interestingly, however, there is a $\sim 10$-fold decrease in $k_{\text {cat }} / K_{M}$ for the ER25 peptide (Supplementary Table S4), further emphasizing that modifications of this residue can fine-tune the substrate specificity of PRMT1.

Photo-Cross-Linking of the PRMT1(Y291pBpF) Mutant and Histones. To verify that Tyr291 plays a role in substrate binding, we first determined whether the $\mathrm{Y} 291 \mathrm{pBpF}$ mutant can cross-link with histone $\mathrm{H} 4$, a known substrate. Histone $\mathrm{H} 3$, which is a relatively poorer PRMT1 substrate, was used as a control (Figure 3C). As expected, histone H4, and to a lesser extent histone $\mathrm{H} 3$, cross-link to the $\mathrm{Y} 291 \mathrm{pBpF}$ mutant. Interestingly, UV irradiation also induces a small amount of cross-linking between the wild type enzyme and histone $\mathrm{H} 4$. Although the source of this cross-linking is unknown, it may relate to the presence of SAM, which is known to photo-crosslink to PRMT $1^{10}$ and other SAM-dependent methyltransferases. $^{17}$

Photo-Cross-Linking and Co-immunoprecipitation of K562 Extract Proteins with the PRMT1(Y291pBpF) Mutant. Having demonstrated the UV-induced cross-linking of $\mathrm{Y} 291 \mathrm{pBpF}$ to a known substrate, we next identified additional interacting proteins, reasoning they would be either substrates or regulatory proteins that modulate the activity and/or substrate specificity of PRMT1 in a phosphorylationdependent manner. Figure 4A represents a schematic depiction of our approach. To first determine the extent of cross-linking, K562 cell extracts were incubated with PRMT1 or the $\mathrm{Y} 291 \mathrm{pBpF}$ mutant and subjected to UV irradiation. K562 cells were used because Tyr291 is phosphorylated in this 


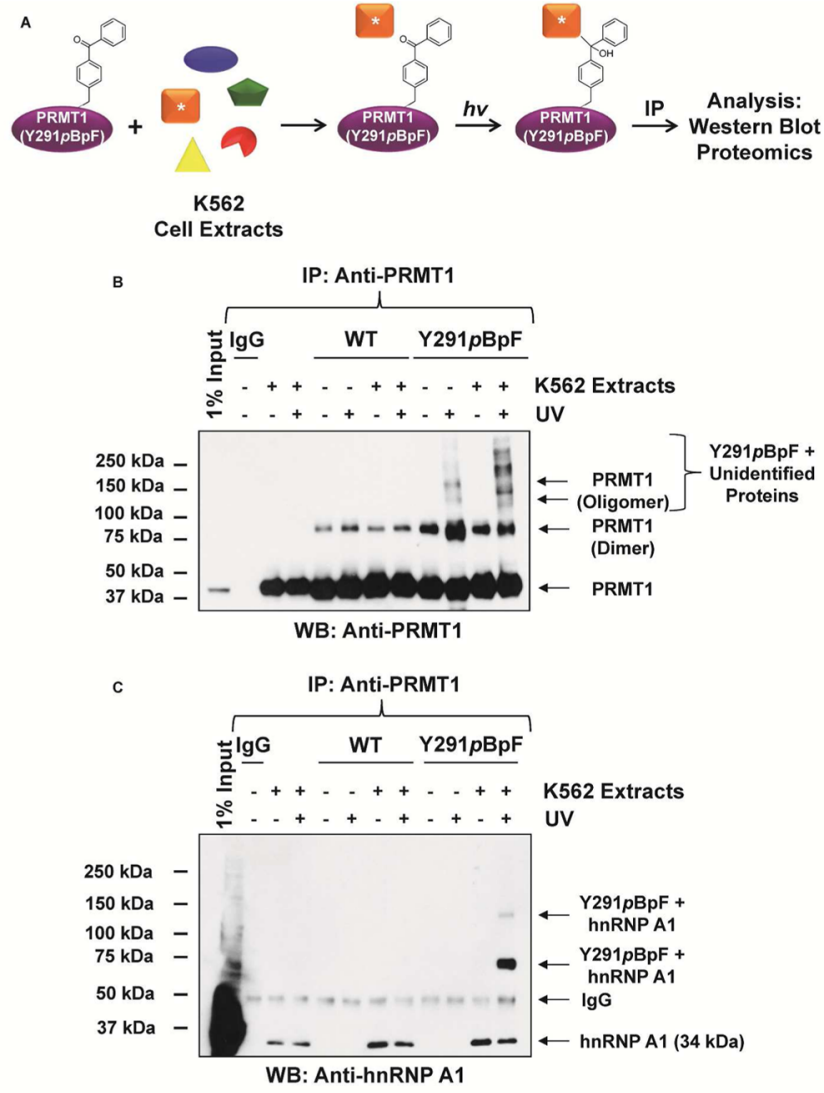

Figure 4. Cross-linking of the PRMT1(Y291pBpF) mutant with K562 cell extracts. (A) PRMT1 cross-linking schematic. (B) Wild type PRMT1 or Y $291 p \mathrm{BpF}$ mutant $(10 \mu \mathrm{g})$ were incubated in the absence or presence of K562 cell extracts $(4.5 \mathrm{mg}$ ) followed by UV irradiation, immunoprecipitation with anti-PRMT1, and Western blot analysis with anti-PRMT1. Significant cross-linking was observed at a high molecular weight between the $\mathrm{Y} 291 \mathrm{pBpF}$ mutant and proteins from the K562 extracts. (C) The Y291pBpF mutant, but not wild type PRMT1, cross-links with hnRNP A1.

myelogenous leukemia-derived cell line. ${ }^{11}$ Protein cross-linking was then confirmed by Western blotting. The results show a significant degree of cross-linking above $75 \mathrm{kDa}$ for the UVtreated samples incubated with the $\mathrm{Y} 291 \mathrm{pBpF}$ mutant (Figure 4B).

To identify these proteins and other PRMT1 interacting proteins, the same procedure was used, except proteins $>75$ $\mathrm{kDa}$ (the approximate size of the PRMT1 dimer) were excised from the gels and subjected to tryptic digestion followed by MS/MS analysis. The results of two independent experiments identified more than 75 proteins that co-immunoprecipitate and/or cross-link to endogenous PRMT1, recombinant PRMT1, and/or the Y291pBpF mutant (Supplementary Table S6). Interestingly, the majority of these proteins are involved in RNA processing and transcription, consistent with the known cellular roles of PRMT1 (Figure 5 and Supplementary Table S7). Importantly, a subset ( 25\%) of the proteins that cross-link with $\mathrm{Y} 291 \mathrm{pBpF}$ are known substrates (e.g., hnRNP A1 and hnRNP H3), confirming a role for this residue in modulating the substrate specificity of PRMT1 (Figure 5 and Supplementary Tables S6-S7). The remaining proteins either represent novel PRMT1 substrates or interacting proteins that regulate PRMT1 activity, substrate specificity, or subcellular localization.

To confirm these interactions, the UV cross-linking experiments were repeated, and the blots were probed with either anti-hnRNP A1 (Figure 4C) or anti-hnRNP H3 antibodies (Supplementary Figure S4B). Gratifyingly, both hnRNP Al and hnRNP H3 cross-link with the Y291pBpF mutant. Further validating these proteins as PRMT1 interacting proteins is the fact that they also co-immunoprecipitate with PRMT1 and the $\mathrm{Y} 291 \mathrm{pBpF}$ mutant in the absence of UV. In total, these data demonstrate that Tyr291 acts as a key point of contact between PRMT1 and its substrates and/or interacting partners.

In Vitro Co-immunoprecipitation and Methylation of hnRNP A1 and hnRNP H3 with the PRMT1(Y291pCmF) Mutant. To evaluate whether Tyr291 phosphorylation alters the interaction between hnRNP A1 and hnRNP $\mathrm{H} 3$, we immunoprecipitated these proteins from K562 cell extracts and then used them in an in vitro co-immunoprecipitation assay with wild type PRMT1 and the Y291 $p \mathrm{CmF}$ mutant. The results of these studies (Figure 6A) indicate that, relative to wild type PRMT1, significantly smaller amounts of the Y291pCmF mutant are co-immunoprecipitated by hnRNP Al and, albeit to a lesser extent, hnRNP H3. On the basis of these results, we conclude that Tyr291 phosphorylation can inhibit interactions between PRMT1 and both hnRNP Al and hnRNP H3. To follow up, we examined the effect of Tyr291 phosphorylation on the rates of hnRNP Al and hnRNP H3 methylation. Immunoprecipitated hnRNP Al and hnRNP $\mathrm{H} 3$ were incubated in the presence of ${ }^{14} \mathrm{C}$-labeled SAM and either PRMT1 or the Y291 $p \mathrm{CmF}$ mutant. The results show that both hnRNP A1 and hnRNP $\mathrm{H} 3$ are poorly methylated by the Y291 $p$ CmF mutant (Figure 6B), thereby demonstrating that Tyr291 phosphorylation alters substrate recognition.

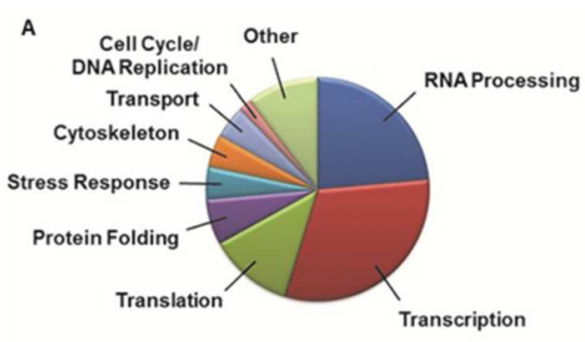

PRMT1 Interacting Proteins

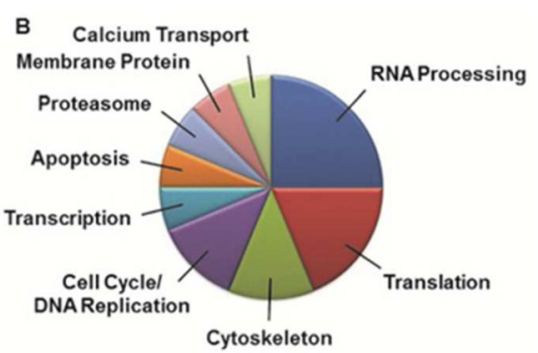

Y291pBpF Crosslinked Proteins

Figure 5. Functions of identified proteins. (A) Functions of proteins that co-immunoprecipitate with both wild type PRMT1 and the Y291pBpF mutant. The majority of the proteins are involved in RNA processing and transcription. (B) Functions of proteins that interact with and/or crosslink to only the $\mathrm{Y} 291 \mathrm{pBpF}$ mutant. 
A

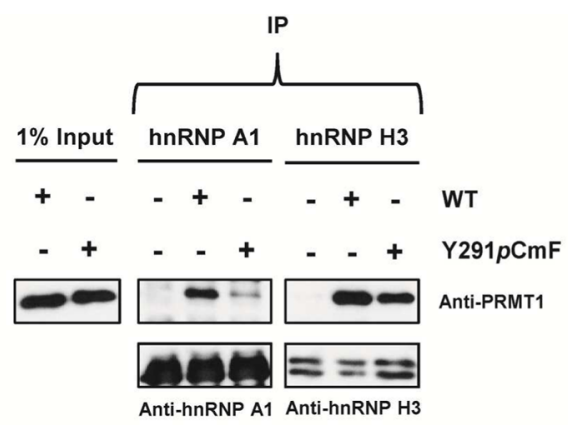

B
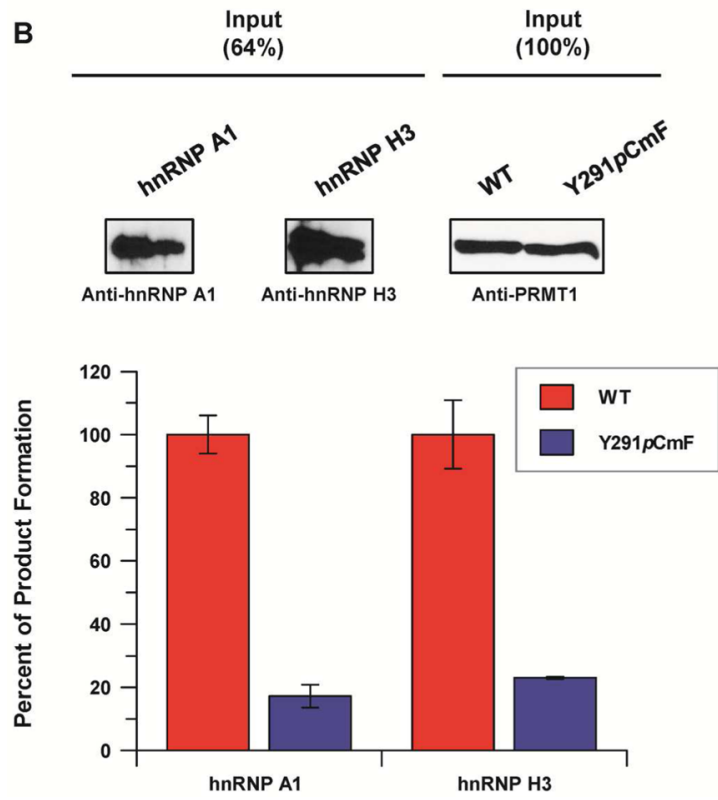

Figure 6. In vitro co-immunoprecipitation and activity assay with hnRNPs. (A) hnRNP Al and hnRNP H3 were immunoprecipitated from K562 cell extracts followed by incubation with either recombinant wild type PRMT1 or the Y $291 p \mathrm{CmF}$ mutant in an in vitro co-immunoprecipitation assay. The samples were analyzed by Western blotting. (B) Immunoprecipitated hnRNP Al or hnRNP H3 were incubated in the presence of wild type PRMT1 or the Y291 $p \mathrm{CmF}$ mutant and ${ }^{14} \mathrm{C}$-labeled SAM followed by phosphorimage analysis.

Conclusions. Taken together, these studies indicate that Tyr291 phosphorylation alters both protein-protein interactions and the substrate specificity of PRMT1 in a substratedependent manner. Since PRMT1 splice variants primarily differ at the $\mathrm{N}$-terminus of the protein and are also important for substrate specificity, ${ }^{18}$ it is possible that phosphorylation of Tyr291 will differentially modulate the substrate specificity of these variants by altering the position of the $\mathrm{N}$-terminal tail. It is also possible that phosphorylation of this residue has a greater effect in vivo, in that the $p \mathrm{CmF}$ residue is still an imperfect mimic of a phosphotyrosine as it differs in both charge and steric bulk. This notion is supported by the fact that the $\mathrm{pBpF}$ mutation, although bulkier, did not induce a more dramatic decrease in $k_{\mathrm{cat}} / K_{\mathrm{M}}$ compared to the $\mathrm{Y} 291 \mathrm{pCmF}$ mutant. Also note that Tyr291 phosphorylation may also modulate PRMT1 activity by providing a docking site for $\mathrm{SH} 2$ domain-containing proteins, which bind to phosphorylated tyrosines. These issues will be addressed in follow up experiments.
With regards to the cross-linking studies, the cellular functions of the cross-linked proteins, as well as those proteins that simply co-immunoprecipitated with PRMT1, coincide with the known roles of PRMT1 in the cell. Interestingly, several novel proteins were specifically recognized by Tyr291, thus further suggesting that this residue is important for either PRMT1 substrate specificity or regulating PRMT1 activity. This conclusion is supported by the in vitro co-immunoprecipitation experiments, which clearly demonstrate that phosphorylation inhibits interactions between PRMT1 and hnRNP A1 and hnRNP H3. In addition, the methyltransferase activity of the $\mathrm{Y} 291 \mathrm{pCmF}$ mutant toward these two proteins was significantly decreased. In total, we show for the first time that phosphorylation of PRMT1 alters protein-protein interactions and substrate specificity.

\section{METHODS}

Site-Directed Mutagenesis. PRMT1 mutants (i.e., Y291F, Y291E, and Y291TAG) were generated using iProofHigh Fidelity DNA Polymerase (Bio-Rad). The Y291F and Y291E mutant plasmids were transformed into E. coli BL21(DE3) cells and purified according to our already established protocol for wild type PRMT1. ${ }^{19}$ The sequence of the Y291F, Y291E and Y291(TAG) mutagenic primers (forward direction only) are 5'-CAGTCCTGAGTCTCCATTCACACATTGGAAGCAG-3'， 5'-CCAGTCCTGAGTCTCCAGAGACACATTGGAAGCAG-3', and 5'-CCAGTCCTGAGTCTCCATAGACACATTGGAAGCAGAC-3', respectively.

Incorporation of $p$-Carboxymethyl-L-phenylalanine $(p \mathrm{CmF})$ and $p$-Benzoyl-L-phenylalanine ( $p \mathrm{BpF})$. Unnatural amino acids were incorporated using methods adapted from previously reported protocols. ${ }^{12,14}$ Briefly, the pEVOL- $p$ CmF or pEVOL- $p$ BpF plasmids were cotransformed into E. coli C43(DE3) cells (Lucigen) with the Y291TAG mutant. Single colonies was used to inoculate starter cultures containing $50 \mu \mathrm{g} / \mathrm{mL}$ kanamycin and $25 \mu \mathrm{g} / \mathrm{mL}$ chloramphenicol in $2 \mathrm{YT}$ media, which were then used to start large scale growths $(1-2 \mathrm{~L})$. These cells were grown at $37{ }^{\circ} \mathrm{C}$ and $200 \mathrm{rpm}$ until $\mathrm{OD}_{600}=0.8$ or 0.5 for the incorporation of $p \mathrm{CmF}$ and $p \mathrm{BpF}$, respectively. The cells were then supplemented with either $1 \mathrm{mM}$ $p \mathrm{CmF}$ or $250 \mu \mathrm{M} p \mathrm{BpF}$, and protein expression was induced with $0.2 \%$ arabinose and isopropyl- $\beta$-D-thiogalactopyranoside ( 1 and 0.4 $\mathrm{mM}$ for the incorporation of $p \mathrm{CmF}$ and $p \mathrm{BpF}$, respectively). The cells were then incubated with shaking at $30{ }^{\circ} \mathrm{C}$ overnight, and the mutant protein was purified according to a protocol already established for wild type PRMT1. ${ }^{19}$

Cell Culture Maintenance and Whole Cell Extract Preparation. K562 cells were grown in Iscove's Modified Dulbecco's Medium (IMDM) (ATCC) supplemented with $10 \%$ fetal bovine serum and $1 \%$ antibiotic/antimycotic solution (Mediatech Inc.) at $37{ }^{\circ} \mathrm{C}$ and $5 \%$ $\mathrm{CO}_{2}$. Cells were removed and centrifuged for $5 \mathrm{~min}$ at $225 \mathrm{~g}$ and $4{ }^{\circ} \mathrm{C}$. After three washes with PBS, cells were resuspended in PBS $(465 \mu \mathrm{L})$ plus complete protease inhibitor cocktail (Roche 4693132) and phosphatase inhibitor cocktail II (Sigma P8465) followed by 75 cycles of sonication ( $1 \mathrm{~s}$ pulse with $10 \mathrm{~s}$ rest at $50 \%$ amplitude). Lysed cells were centrifuged for $5 \mathrm{~min}$ at $3824 \mathrm{~g}$ at $4{ }^{\circ} \mathrm{C}$, and the supernatant was removed as the whole cell extract. Protein concentration was determined by a DC Protein Assay (Bio-Rad).

UV Cross-Linking with Histone $\mathrm{H} 3$ and H4. Wild type PRMT1 or the $\mathrm{Y} 291 \mathrm{pBpF}$ mutant $(0.5 \mu \mathrm{g})$ was incubated for $10 \mathrm{~min}$ on ice in the absence and presence of histone $\mathrm{H} 3$ and $\mathrm{H} 4(1 \mu \mathrm{g})$ individually. Samples were then subjected to $30 \mathrm{~min}$ of irradiation with UV light $(365 \mathrm{~nm})$ on ice in a 96-well plate. Both the UV-treated samples and control samples (non-UV-treated) were quenched with SDS-loading dye and analyzed by Western blotting (see Supplementary Methods).

UV Cross-Linking and Immunoprecipitation with K562 Cell Extracts. Wild type PRMT1 or the $\mathrm{Y} 291 \mathrm{pBpF}$ mutant was incubated for $10 \mathrm{~min}$ on ice in the absence and presence of K562 cell extracts $(4.5 \mathrm{mg})$ plus complete protease inhibitor cocktail (Roche-4693132). Two independent experiments were performed, using either 45 or 10 
$\mu \mathrm{g}$ of enzyme. PRMT1 samples, plus K562 cell extracts alone, were then subjected to $30 \mathrm{~min}$ of irradiation with UV light $(365 \mathrm{~nm})$ on ice in a 96-well plate. Both the UV-treated samples and control samples (non-UV-treated) were incubated with rotation for $1 \mathrm{~h}$ with antiPRMT1 (Bethyl A300-722A) at $4{ }^{\circ} \mathrm{C}$. Protein A/G Plus (sc-2003) (20 $\mu \mathrm{L}$ ) was then added, and samples were incubated overnight at $4{ }^{\circ} \mathrm{C}$ with rotation. Beads were collected by centrifugation at $1593 \mathrm{~g}$ for 2 min and washed $3 \times$ with $200 \mu \mathrm{L}$ of cold PBS. The supernatant was removed, and $30 \mu \mathrm{L}$ of $1 \times$ SDS-loading buffer was added followed by heating at $95{ }^{\circ} \mathrm{C}$ for $10 \mathrm{~min}$. Beads were collected by centrifugation at $1593 \mathrm{~g}$ for $2 \mathrm{~min}$, and the supernatant was removed and placed in a new tube. Samples $(15 \mu \mathrm{L})$ were then separated by $12 \%$ SDS-PAGE. Additionally, $2-\mu \mathrm{L}$ aliquots of the heated samples were removed followed by the addition of $10 \mu \mathrm{L}$ of $1 \times$ SDS-loading buffer to each to be used for Western blot analysis. Proteins were identified by MS/MS analysis (see Supplementary Methods for details).

In Vitro Co-immunoprecipitation with Wild Type and the Y291pCmF Mutant. K562 cell extracts $(4.5 \mathrm{mg}$ ) plus complete protease inhibitor cocktail (Roche 4693132) were incubated with rotation for $1 \mathrm{~h}$ with anti-hnRNP Al (Santa Cruz sc-32301) or antihnRNP H3 (Santa Cruz sc-376416) antibodies at $4{ }^{\circ} \mathrm{C}$. Protein A/G Plus (sc-2003) $(30 \mu \mathrm{L})$ was then added, and samples were incubated overnight at $4{ }^{\circ} \mathrm{C}$ with rotation. Beads were collected by centrifugation at $1593 \mathrm{~g}$ for $2 \mathrm{~min}$ and washed $3 \times$ with $200 \mu \mathrm{L}$ of cold PBS. Wild type or the $\mathrm{Y} 291 p \mathrm{CmF}$ mutant $(10 \mu \mathrm{g})$ in cold PBS ( $250 \mu \mathrm{L}$ final volume) was added, and samples were incubated with rotation for $1 \mathrm{~h}$. Beads were collected by centrifugation at $1593 g$ for $2 \mathrm{~min}$ and washed $3 \times$ with $200 \mu \mathrm{L}$ of cold PBS. The supernatant was removed and $50 \mu \mathrm{L}$ of $1 \times$ SDS-loading buffer was added followed by heating at $95{ }^{\circ} \mathrm{C}$ for 10 min. Beads were collected by centrifugation at $1593 \mathrm{~g}$ for $2 \mathrm{~min}$, and the supernatant was removed and placed in a new tube. Samples ( 9 $\mu \mathrm{L}$ ) were then separated by $12 \%$ SDS-PAGE and visualized by Western blot analysis.

In Vitro Methylation of Immunoprecipitated hnRNPs. K562 cell extracts $(6 \mathrm{mg})$ plus complete protease inhibitor cocktail (Roche 4693132) were incubated with rotation for $1 \mathrm{~h}$ with anti-hnRNP Al (Santa Cruz sc-32301) or anti-hnRNP H3 (Santa Cruz sc-376416), three separate samples per antibody, at $4{ }^{\circ} \mathrm{C}$. Protein A/G Plus (sc2003) $(30 \mu \mathrm{L})$ was then added, and samples were incubated overnight at $4{ }^{\circ} \mathrm{C}$ with rotation. Beads were collected by centrifugation at $1593 \mathrm{~g}$ for $2 \mathrm{~min}$ and washed $3 \times$ with $200 \mu \mathrm{L}$ of cold PBS. Beads were incubated for $10 \mathrm{~min}$ at RT with $50 \mu \mathrm{L}$ of $100 \mathrm{mM}$ glycine- $\mathrm{HCl} \mathrm{pH}$ 2.7 followed by centrifugation at $1593 \mathrm{~g}$ for $2 \mathrm{~min}$. The supernatant was removed and neutralized with $5 \mu \mathrm{L}$ of $1 \mathrm{M}$ HEPES pH 8.2. Beads were then incubated a second time for $10 \mathrm{~min}$ at RT with $50 \mu \mathrm{L}$ of $100 \mathrm{mM}$ glycine- $\mathrm{HCl} \mathrm{pH} 2.7$ followed by centrifugation at $1593 \mathrm{~g}$ for $2 \mathrm{~min}$. The supernatant was removed and combined with previous supernatant and neutralized with $5 \mu \mathrm{L}$ of $1 \mathrm{M}$ HEPES $\mathrm{pH}$ 8.2. The supernatants from three separate samples for each antibody were combined and concentrated using Amicon Ultra-0.5 Centrifugal Filter Devices 10,000 NMWL (UFC501096) for $5 \mathrm{~min}$ at 16,000g. Utilizing the radioactive based assay described above, reaction mixtures of $50 \mathrm{mM}$ HEPES $\mathrm{pH}$ 8.0, $1 \mathrm{mM}$ EDTA, $50 \mathrm{mM} \mathrm{NaCl}, 0.5 \mathrm{mM}$ dithiothreitol, $15 \mu \mathrm{M}\left[{ }^{14} \mathrm{C}\right]$ labeled SAM, and $14 \mu \mathrm{L}$ of immunoprecipitated hnRNP A1 or hnRNP $\mathrm{H} 3,27 \mu \mathrm{L}$ total reaction volume, were preincubated at $37{ }^{\circ} \mathrm{C}$ for 10 min. After the addition of $3 \mu \mathrm{L}$ of $50 \mathrm{mM}$ HEPES (Control), wild type PRMT1 $(2 \mu \mathrm{M})$, or the $\mathrm{Y} 291 p \mathrm{CmF}$ mutant $(2 \mu \mathrm{M})$, the reaction was allowed to run for $1 \mathrm{~h}$ before being quenched with $6 \times$ SDS gel loading buffer. Each assay was done in duplicate. Samples $(15 \mu \mathrm{L})$ were separated by $12 \%$ SDS-PAGE followed by visualization and quantification using phosphorimage analysis (Molecular Dynamics). For Western blot input controls, $9 \mu \mathrm{L}$ of concentrated immunoprecipitated hnRNP A1 and hnRNP H3, as well as $200 \mathrm{nM}$ wild type PRMT1 and the Y291 $p$ CmF mutant, was used and analyzed according to the Western blot protocol described in the Supporting Information.

\section{ASSOCIATED CONTENT}

\section{Supporting Information}

Supplemental methods for the synthesis of $p$-carboxymethyl-Lphenylalanine $(p \mathrm{CmF})$, in-gel digest and MS/MS analysis of Y291 $p$ CmF mutant and UV cross-linking samples, purification of histone H4, peptide synthesis, gel-based methylation assay, MALDI-MS based methylation assay, Western blotting, and partial proteolysis. Supplemental scheme, figures, and tables. This material is available free of charge via the Internet at http://pubs.acs.org.

\section{AUTHOR INFORMATION}

\section{Corresponding Author}

*E-mail: pthompso@scripps.edu.

Notes

The authors declare no competing financial interest.

\section{ACKNOWLEDGMENTS}

We thank P. Martínez-Acedo for his gracious help confirming the incorporation of $p \mathrm{CmF}$ into PRMT1. This work was supported in part by funding provided to P.R.T. by TSRI.

\section{ABBREVIATIONS}

PRMT, protein arginine methyltransferases; $p \mathrm{CmF}, p$-carboxymethyl-L-phenylanine; $p \mathrm{BpF}, p$-benzoyl-L-phenylalanine; PTMs, post-translational modifications; SAM, S-adenosylmethionine; ADMA, asymmetrically dimethylated arginine; UAA, unnatural amino acid; hnRNP, heterogeneous ribonucleoprotein

\section{REFERENCES}

(1) Bedford, M. T., and Richard, S. (2005) Arginine methylation an emerging regulator of protein function. Mol. Cell 18, 263-272.

(2) Bedford, M. T., and Clarke, S. G. (2009) Protein arginine methylation in mammals: who, what, and why. Mol. Cell 33, 1-13.

(3) Feng, Q., He, B., Jung, S. Y., Song, Y., Qin, J., Tsai, S. Y., Tsai, M. J., and O'Malley, B. W. (2009) Biochemical control of CARM1 enzymatic activity by phosphorylation. J. Biol. Chem. 284, 3616736174.

(4) Higashimoto, K., Kuhn, P., Desai, D., Cheng, X., and Xu, W. (2007) Phosphorylation-mediated inactivation of coactivator-associated arginine methyltransferase 1. Proc. Natl. Acad. Sci. U.S.A. 104, 12318-12323.

(5) Carascossa, S., Dudek, P., Cenni, B., Briand, P. A., and Picard, D. (2010) CARM1 mediates the ligand-independent and tamoxifenresistant activation of the estrogen receptor alpha by cAMP. Genes Dev. 24, 708-719.

(6) Liu, F., Zhao, X., Perna, F., Wang, L., Koppikar, P., Abdel-Wahab, O., Harr, M. W., Levine, R. L., Xu, H., Tefferi, A., Deblasio, A., Hatlen, M., Menendez, S., and Nimer, S. D. (2011) JAK2V617F-mediated phosphorylation of PRMT5 downregulates its methyltransferase activity and promotes myeloproliferation. Cancer Cell 19, 283-294.

(7) Yue, W. W., Hassler, M., Roe, S. M., Thompson-Vale, V., and Pearl, L. H. (2007) Insights into histone code syntax from structural and biochemical studies of CARM1 methyltransferase. EMBO J. 26, $4402-4412$.

(8) Troffer-Charlier, N., Cura, V., Hassenboehler, P., Moras, D., and Cavarelli, J. (2007) Functional insights from structures of coactivatorassociated arginine methyltransferase 1 domains. EMBO J. 26, 43914401.

(9) Rust, H. L., Zurita-Lopez, C. I., Clarke, S., and Thompson, P. R. (2011) Mechanistic studies on transcriptional coactivator protein arginine methyltransferase 1 . Biochemistry 50, 3332-3345.

(10) Tang, J., Frankel, A., Cook, R. J., Kim, S., Paik, W. K., Williams, K. R., Clarke, S., and Herschman, H. R. (2000) PRMT1 is the 
predominant type I protein arginine methyltransferase in mammalian cells. J. Biol. Chem. 275, 7723-7730.

(11) Hornbeck, P. V., Kornhauser, J. M., Tkachev, S., Zhang, B., Skrzypek, E., Murray, B., Latham, V., and Sullivan, M. (2012) PhosphoSitePlus: a comprehensive resource for investigating the structure and function of experimentally determined post-translational modifications in man and mouse. Nucleic Acids Res. 40, D261-270.

(12) Xie, J., Supekova, L., and Schultz, P. G. (2007) A genetically encoded metabolically stable analogue of phosphotyrosine in Escherichia coli. ACS Chem. Biol. 2, 474-478.

(13) Szewczuk, L. M., Tarrant, M. K., and Cole, P. A. (2009) Protein phosphorylation by semisynthesis: from paper to practice. Methods Enzymol 462, 1-24.

(14) Young, T. S., Ahmad, I., Yin, J. A., and Schultz, P. G. (2010) An enhanced system for unnatural amino acid mutagenesis in E. coli. J. Mol. Biol. 395, 361-374.

(15) Kauer, J. C., Erickson-Viitanen, S., Wolfe, H. R., Jr., and DeGrado, W. F. (1986) p-Benzoyl-L-phenylalanine, a new photoreactive amino acid. Photolabeling of calmodulin with a synthetic calmodulin-binding peptide. J. Biol. Chem. 261, 10695-10700.

(16) Chin, J. W., Martin, A. B., King, D. S., Wang, L., and Schultz, P. G. (2002) Addition of a photocrosslinking amino acid to the genetic code of Escherichia coli. Proc. Natl. Acad. Sci. U.S.A. 99, 11020-11024.

(17) Wenzel, C., Moulard, M., Lobner-Olesen, A., and Guschlbauer, W. (1991) Crosslinking of Dam methyltransferase with S-adenosylmethionine. FEBS Lett. 280, 147-151.

(18) Goulet, I., Gauvin, G., Boisvenue, S., and Cote, J. (2007) Alternative splicing yields protein arginine methyltransferase 1 isoforms with distinct activity, substrate specificity, and subcellular localization. J. Biol. Chem. 282, 33009-33021.

(19) Osborne, T. C., Obianyo, O., Zhang, X., Cheng, X., and Thompson, P. R. (2007) Protein arginine methyltransferase 1: positively charged residues in substrate peptides distal to the site of methylation are important for substrate binding and catalysis. Biochemistry 46, 13370-13381. 